\title{
Isoflavones and biotransformed dihydrodaidzein in hairy roots of Korean wild arrowroot
}

\author{
Eunji Lee $\cdot$ Tae-Ho Park
}

Received: 16 January 2016 / Revised: 18 February 2016 / Accepted: 21 February 2016

(c) Korean Society for Plant Biotechnology

\begin{abstract}
Pueraria lobata is a perennial legume plant that produces a variety of isoflavones, such as puerarin, daidzin and daidzein. These are metabolized to equol via dihydrodaidzein and tetrahydrodaidzein by the bacterial fermentation of natural isoflavone sources in the human intestines. In this study, we described the growth and accumulation of isoflavone in the hairy root of the Korean wild arrowroot according to the culture period, as well as dihydrodaidzein biosynthesis in hairy root extracts fermented with Pediococcus pentosaceus. Daily proliferation was best in DY1 cultured for 1 week. DY1 showed significant differences in daily production of puerarin and daidzin+daidzein, as compared to DJ7; furthermore, both were best in DY1 cultured for 1 week. The hairy root extract was fermented successfully with $P$. pentosaceus with confirmed production of dihydrodaidzein, an equol precursor formed by biotransformation. The results indicated that the growth of hairy roots and isoflavone accumulation in the hairy roots is best 1 week after culture. These results are expected to contribute to the mass production of hairy root and isoflavones as equol precursors from the Korean wild arrowroot and provide a basis for equol production by biotransformation in vitro.
\end{abstract}

Keywords Biotransformation, Dihydrodaidzein, Isoflavone, Hairy root, Pediococcus pentosaceus, Pueraria lobata

\section{Introduction}

Pueraria lobata, which has the common names, arrowroot, kudzu and chik, is a perennial leguminous plant that is

\footnotetext{
E. Lee $\cdot$ T.-H. Park $(\bowtie)$

Department of Horticulture and Institute of Life and Environment, Daegu University, Gyeongsan 38453, Republic of Korea

e-mail: thzoo@daegu.ac.kr
}

distributed widely in tropical and subtropical countries of East Asia and is one of the oldest medicinal plants in Korea, China and Japan (Chen and Li 2007; Kim et al. 2009).

These plants have been used extensively for the treatment of migraine, tonsillitis and hypertension, and as an antispasmodic, antipyretic and diaphoretic (Yuk 1989). In addition, they have a similar role to phytoestrogens, which have potent biological activity that might reduce the risk of osteoporosis, cardiovascular disorders and menopausal disorders (Cherdshewasart et al. 2007; Watanabe et al. 2000). The major chemical constituents in the tubers of $P$. lobata are isoflavones, including puerarin, daidzin, genistin, daidzein, and genistein (Chansakaow et al. 2000). These isoflavones exist predominantly in legume plants, such as soybean, and particularly many research results using soybean were reported (Barnes 1998; Cassidy 1996; Setchell 1998; Setchell et al. 2013). Although the genus Pueraria has been known to contain more isoflavones than soybean (Kaufman et al. 1997), there have been relatively few studies of the genus Pueraria. Moreover, after the ingestion of soy-products, equol is a metabolite produced by biotransformation. Although equol has stronger estrogenic activity than other isoflavone derivatives (Sathyamoorthy and Wang 1997; Setchell et al. 2002), it is well known that approximately $30 \sim 50 \%$ of healthy human is able to produce equol (Arai et al. 2000). The reason for this is the individual differences existing in the ability of the intestinal microflora to produce equol (Arai et al. 2000; Setchell et al. 2002). Equol production is not a metabolism of plant origin and is entirely a metabolic product converted from isoflavones via an intestinal bacterial metabolism. In addition, its production may rely on the existence of bacterial species capable of metabolizing daidzein, which is one of the precursors of equol among isoflavones (Decroos et al. 2005; Setchell et al. 2002). Recently, several bacteria capable of producing equol have been isolated from the feces of humans or animals, such as rats and pigs (Minamida et al. 2006; Yokoyama and Suzuki 2008; Yu et 
al. 2008). On the other hand, the metabolism pathway produced equol is unclear. An investigation of the enzymes in recent studies reported the Lactococcus and Slakia strain could convert daidzein to dihydrodaidzein (DHD), and the dihydrodaidzein produced could be metabolized to equol via tetrahydrodaidzein (THD) (Shimada et al. 2010; Tsuji et al 2012). The pathway before daidzein suggested that puerarin and daidzin were converted to daidzein (Jin et al. 2008; Park et al. 2006).

In previous studies, the possibility of the enhanced accumulation of isoflavones has been reported in cell suspension cultures (Boonsnongcheep et al. 2010; Korsangruang et al. 2010; Lee et al. 2013; Vaishnav et al. 2006). On the other hand, there are only a few reports on hairy root cultures, despite isoflavone production by hairy root cultures being studied in a Psoralea species and other Pueraria species (Bourgaud et al. 1999; Udomsuk et al. 2011). Therefore, this study examined hairy root growth and the level of isoflavones production in the hairy root cultures of Korean wild arrowroot. In addition, this study examined whether dihydrodaidzein as a precursor of equol is produced in the hairy root extracts fermented with Pediococcus pentosaceus and whether equol can be produced by biotransformation in vitro.

\section{Materials and Methods}

Collection of plant materials and hairy root growth

Eighteen different lines of the Korean wild arrowroot, $P$. lobata, were collected in four different regions, Danyang, Mungyeong, Daejeon and Gyeongsan, in early spring before sprouting (Table 1). They were planted in plastic pots and maintained in a greenhouse at Daegu University. When the plants sprouted, the stem segments were dissected, sterilized and placed in vitro for the induction and production of hairy roots, as described by Kim et al. (2012). For the induction and subculture of hairy roots, a 1/2 MS medium (Murashige and Skoog 1962) containing $0.5 \mathrm{mg} / \mathrm{L}$ indole-3-butyric acid (IBA) was used. All explants and hairy roots were cultured in the dark and the hairy roots induced were subcultured on fresh medium every two weeks.

To examine the growth of hairy roots and the accumulation of isoflavones in vitro, two lines (DJ7 and DY1) of the collected arrowroots were selected. The fresh hairy roots $(1 \mathrm{~g}$ each) of the two lines cultured on static medium were excised and cultured in a $250 \mathrm{ml}$ Erlenmeyer flask containing $100 \mathrm{ml}$ of $1 / 2 \mathrm{MS}$ liquid medium with $0.5 \mathrm{mg} / \mathrm{L}$ IBA for one to four weeks. The hairy roots were harvested each week $(1,2,3$ and 4 weeks after the initial culture) and their fresh weight (FW)
Table 1 Plant materials collected from the 4 different regions and their puerarin and daidzin contents

\begin{tabular}{ccccc}
\hline Name & Character $^{\mathrm{z}}$ & Region $^{\mathrm{y}}$ & Puerarin $^{\mathrm{x}}$ & Daidzin $^{\mathrm{x}}$ \\
\hline DY1 & Amchik & Danyang & $\mathrm{nd}^{\mathrm{w}}$ & nd \\
DY2 & Amchik & Danyang & nd & nd \\
DY3 & Suchik & Danyang & 1.96 & 0.27 \\
DY4 & Suchik & Danyang & nd & nd \\
MG1 & Suchik & Mungyeong & 1.17 & 0.72 \\
MG2 & Suchik & Mungyeong & 1.44 & 0.77 \\
MG3 & Suchik & Mungyeong & 1.04 & 0.89 \\
MG4 & Amchik & Mungyeong & 1.12 & 0.83 \\
MG5 & Amchik & Mungyeong & 0.68 & 0.52 \\
DJ1 & Suchik & Daejeon & 1.57 & 0.37 \\
DJ2 & Suchik & Daejeon & 1.60 & 0.65 \\
DJ3 & Suchik & Daejeon & 1.78 & 0.59 \\
DJ4 & Amchik & Daejeon & 0.78 & 0.57 \\
DJ5 & Amchik & Daejeon & 1.95 & 0.99 \\
DJ6 & Amchik & Daejeon & 1.33 & 0.72 \\
DJ7 & Amchik & Daejeon & 0.66 & 0.27 \\
GS1 & Amchik & Gyeongsan & nd & nd \\
GS2 & Amchik & Gyeongsan & nd & nd \\
\hline
\end{tabular}

${ }^{\mathrm{z}}$ Two types of $P$. lobata, such as male and female arrowroot (preferably renamed Suchik and Amchik as Korean wild species of $P$. lobata, respectively).

${ }^{y}$ Geographic regions in which tubers of $P$. lobata were collected. ${ }^{\mathrm{x}}$ Puerarin and daidzin content are given in milligram per gram dry weight.

${ }^{\mathrm{w}}$ Not determined

was measured. Subsequently, they were freeze-dried and their dry weight (DW) was also measured. The experiment was conducted with six replications.

Isoflavones extraction and analysis

The tubers of thirteen lines were used to analyze the isoflavones (puerarin and daidzin) in the tubers of the collected Korean wild arrowroot. The hairy roots were also analyzed to examine the ability of isoflavone accumulation in vitro depending on the culture period. After measuring the dry weight, as mentioned above, $20 \%$ ethanol extracts from the hairy roots were prepared and the isoflavone (puerarin and daidzin+daidzein) contents were analyzed.

The following reagents were used as standard substances: puerarin (P5555 Sigma-Aldrich, MG, USA), daidzin (30408 Sigma-Aldrich, MG, USA), daidzein (D7802 Sigma-Aldrich, MG, USA) and dihydrodaidzein (SC-17238-05-0 Santacruz, CA, USA). An SIL-20A equipped with a SPD-M20A PDA and CTO-20A column oven (SHIMADZU Corp., Kyoto, 
Japan) was used for HPLC analysis. The data were analyzed using the attached software. The fractions were separated at $40^{\circ} \mathrm{C}$ on an ACE-121-2546 apparatus (Advanced Chromatography Technologies Corp., Aberdeen, Scotland) using a one-step linear gradient. The ratios of the mobile phases A [water : acetic acid (100:1)] and B [water : acetonitrile : acetic acid (50:50:1)] were changed after 5 minutes from 7:3 to $3: 7$ over a 40 minute period at a flow rate of $1.0 \mathrm{~mL} / \mathrm{min}$.

Fermentation of hairy root extracts with Pediococcus pentosaceus

To investigate the alterations of substances according to the fermentation periods of the hairy root extracts of two Korean wild arrowroots (DJ7 and DY1) with P. pentosaceus EP106 (KACC91829P, Genebank Information Center, Rural Development Administration, Korea), the hairy roots were cultured in a $250 \mathrm{ml}$ Erlenmeyer flask containing the above-mentioned medium for 12-14 days. The hairy roots were harvested and freeze-dried. Approximately $2 \mathrm{~g}$ (DW) of each dried sample was collected. The freeze-dried hairy roots were extracted three times with $20 \%$ aqueous ethanol $(\mathrm{EtOH})$ at room temperature. The combined extracts were evaporated to dryness under a reduced pressure, and the residues were suspended in distilled water. Before fermentation, the bacterial cells, P. pentosaceus EP106, were maintained on GAM (consisting of $1 \%$ peptone, $0.3 \%$ soybean peptone, $1 \%$ proteose peptone, $1.35 \%$ digested serum powder, $0.5 \%$ yeast extract, $0.22 \%$ heart extract, $0.12 \%$ liver extract powder, $0.3 \%$ glucose, $0.25 \% \mathrm{KH}_{2} \mathrm{PO}_{4}, 0.3 \% \mathrm{NaCl}, 0.5 \%$ soluble starch, $0.03 \%$ L-cysteine hydrochloride and $0.03 \%$ sodium thoglycolate) agar plates and inoculated into $10 \mathrm{~mL}$ of fresh GAM liquid medium in $50 \mathrm{~mL}$ tubes in a standing incubator at $28^{\circ} \mathrm{C}$ overnight. The cultured cells were washed with GAM medium, and adjusted to an optical density of 1.0 at $600 \mathrm{~nm}$ in GAM liquid medium. The cells were then dispensed into $250 \mathrm{~mL}$ flasks containing $10 \mathrm{~mL}$ of the GAM medium. The hairy root extracts were added to the cultures to a final concentration of $2 \%(\mathrm{w} / \mathrm{v})$, and incubated aerobically $(250 \mathrm{rpm})$ at $28^{\circ} \mathrm{C}$. Subsequently, $1 \mathrm{~mL}$ samples were taken at $0,24,48$ and 72 hr. The puerarin, daidzin, daidzein and dihydordaidzein levels were analyzed from the samples taken using a HPLC analysis system.

\section{Statistical analysis}

The contents of puerarin and daidzin in tubers of 13 arrowroot lines were analyzed using a t-test to confirm the difference of isoflavone accumulation between female and male arrowroots. The fresh and dry weight of the hairy roots and the contents of puerarin and daidzin + daidzein in the hairy roots, which were scored one, two, three, and four weeks after the initial culture, were analyzed using an analysis of the variance (ANOVA). After defining the effects of the culture period, Duncan's multiple-range test (DMRT) was set at $\mathrm{P}<0.05$.

\section{Results and Discussion}

\section{Characteristics of the collected arrowroots}

Initially, 18 lines of Korean wild arrowroot, $P$. lobata (Table 1) were collected from four different national regions to set up the system of hairy root induction and culture that was successfully achieved as described by Kim et al. (2012). The collected arrowroots were assigned to male and female arrowroots from their general characteristic, and are preferably referred Suchik and Amchik, respectively. This is not a botanical distinction (Spennemann 1992, 1994), but it is known that Amchik contains more starch and less fiber than Suchik and bears larger tubers. The original name of Korean wild species $P$. lobata 'Chik' has recently been registered in CODEX and can be used as it is in Korean.

Puerarin and daidzin in the tubers of 13 of the collected lines were analyzed by HPLC. The average contents of puerarin and daidzin were 1.31 and $0.63 \mathrm{mg} / \mathrm{g}$ of dry weight, respectively (Table 1). These results are different from those reported by Junko et al. (1984), Kim and Park (1987) and Oh et al. (1990). The puerarin and/or daidzin contents in this study were lower than those in their studies with the Korean

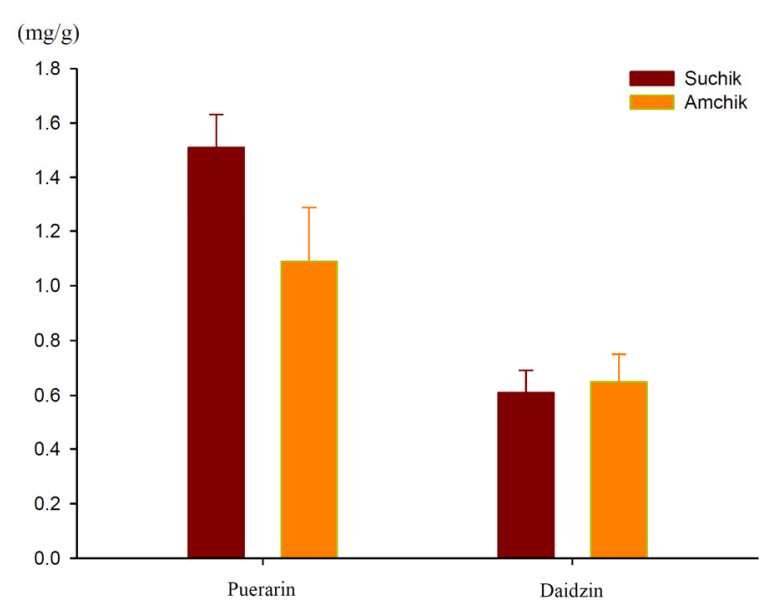

Fig. 1 Puerarin and daidzin contents in the male (Suchik) and female (Amchik) Korean wild arrowroots, P. lobata. The error bars represent the standard error of the means of 6 or 7 replicates (lines) of Amchik and Suchik, respectively 
wild arrowroots. The variation of the contents was reported to be caused by differences in the extraction method, arrowroot line, collection region, growth condition, etc. (Kim and Park 1987; Oh et al. 1990). When the results were analyzed separately as Suchik and Amchik, the average contents of puerarin in Suchik and Amchik were 1.51 and $1.09 \mathrm{mg} / \mathrm{g}$ of dry weight, respectively, and the average contents of daidzin were 0.61 and $0.65 \mathrm{mg} / \mathrm{g}$ of dry weight, respectively. The puerarin content was significantly higher in Suchik than in Amchik, but the daidzin content was not significantly different (Fig. 1). Normally, Suchik has known to produce more functional substances than Amchik even though the latter is more attractive for eating in Korea than the former because of its better taste and chewy texture (personal communication). On the other hand, Shin et al. (2000) reported that the puerarin, daidzin and daidzein contents were higher in Amchik than in Suchik, whereas the antimicrobial activity was higher in Suchik than in Amchik.

Growth of hairy root

Although many results in the growth curves of cell suspension cultures with Pueraria species have been reported (Boons-

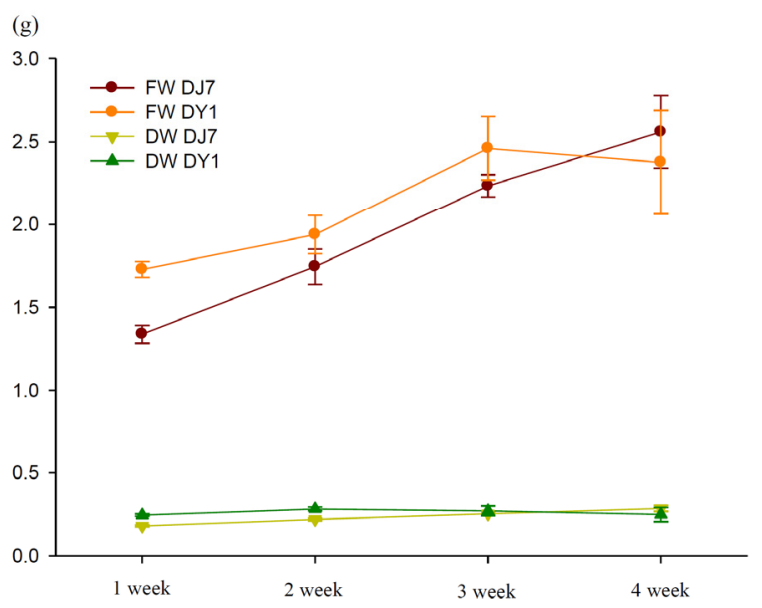

Fig. 2 Growth curves of the hairy root suspension culture scored over the 4 week culture period in 2 different lines (DJ7 and DY1) of the Korean wild arrowroot, P. lobata. FW and DW indicate the fresh and dry weight, respectively. The error bars represent the standard error of the means of 6 replicates nongcheep et al. 2010; Chen and Li 2007; Korsangruang et al. 2010), those of hairy root cultures were rare. Therefore, the growth characteristics of the hairy roots were examined during the 4 weeks of culture using the fresh and dry weights. Figure 2 shows the growth pattern of the hairy root cultures based on the fresh and dried biomass of two different lines (DJ7 and DY1), which were selected from the 18 collected lines because of their relatively vigorous growth in both solid and liquid media. The growth curves and statistical analyses indicated that the growth of hairy roots in DJ7 was not significantly different from that in DY1. On the other hand, both the fresh and dry weight weekly scored for four weeks were significantly different at $P$ values of 0.000002 and 0.047 , respectively. The highest average fresh biomass from all the experiments of DJ7 was $2.56 \mathrm{~g} / \mathrm{flask}$, which occurred on the $4^{\text {th }}$ week of culture and that of DY1 was 2.46 $\mathrm{g} /$ flask on the $3^{\text {rd }}$ week of culture (Fig. 2). The highest mean dried biomass of DJ7 was $285 \mathrm{mg} /$ flask on the $4^{\text {th }}$ week of culture, whereas that of DY1 was $282 \mathrm{mg} /$ flask on the $2^{\text {nd }}$ week of culture (Fig. 2). This suggests that the fresh weight has a tendency to increase gradually with increasing culture period, but the dry weight does not. This shows that the dry weight of Pueraria candollei hairy root culture has a different pattern in the growth curve, which was also reported by Udomsuk et al. (2011). The difference in daily growth of hairy roots between DJ7 and DY1 was significant at $\mathrm{P}<0.05$.

The highest mean daily fresh biomass in DJ7 was 0.059 $\mathrm{g} /$ day on the $3^{\text {rd }}$ week of culture, whereas that of DY1 was $0.104 \mathrm{~g} /$ day on the $1^{\text {st }}$ week of culture (Table 2). When the two lines were analyzed separately, the daily growth of hairy roots measured every week was significantly different in DY1, but not in DJ7. This difference between DJ7 and DY1 might be due to the sensitivity to a prolonged suspension culture and caused by either the loss of the hairy root's capacity for recognizing the stimulus or a loss of competency factors in the hairy root population with time.

Isoflavone accumulation at the cultured hairy roots

The level of isoflavone accumulation was also studied in the hairy roots of $P$. lobata cultured for 4 weeks. The contents of puerarin and daidzin+daidzein in the $20 \%$ ethanol extracts

Table 2 Daily growth of the hairy root of 2 arrowroot lines. The experiment was conducted 6 times over a 4-week period

\begin{tabular}{ccccc}
\hline & 1 week & 2 weeks & 3 weeks & 4 weeks \\
\hline $\mathrm{DJ}^{\mathrm{z}, \mathrm{y}}$ & $0.048 \pm 0.008^{\mathrm{b}}$ & $0.053 \pm 0.008^{\mathrm{b}}$ & $0.059 \pm 0.003^{\mathrm{b}}$ & $0.056 \pm 0.008^{\mathrm{b}}$ \\
$\mathrm{DY} 1^{\mathrm{z} y}$ & $0.104 \pm 0.007^{\mathrm{a}}$ & $0.067 \pm 0.008^{\mathrm{b}}$ & $0.070 \pm 0.009^{\mathrm{b}}$ & $0.049 \pm 0.011^{\mathrm{b}}$ \\
\hline
\end{tabular}

${ }^{\mathrm{z}}$ Average fresh weight of hairy root is given in grams per day.

${ }^{\mathrm{y}}$ The values represent the mean \pm standard error, and the mean values with a different letter are significantly different at $\mathrm{P}<0.05$. 
Table 3 Puerarin contents in the hairy root for 2 arrowroot lines. The experiment was conducted 6 times over a 4 -week period

\begin{tabular}{ccccc}
\hline & 1 week & 2 weeks & 3 weeks & 4 weeks \\
\hline $\mathrm{DJ}^{\mathrm{zy}}$ & $39.0 \pm 2.7^{\mathrm{a}}$ & $33.1 \pm 2.8^{\mathrm{ab}}$ & $34.1 \pm 3.6^{\mathrm{ab}}$ & $37.9 \pm 2.6^{\mathrm{a}}$ \\
$\mathrm{DY}^{\mathrm{z}, \mathrm{y}}$ & $38.0 \pm 1.6^{\mathrm{a}}$ & $40.5 \pm 2.2^{\mathrm{a}}$ & $24.9 \pm 3.9^{\mathrm{bc}}$ & $18.8 \pm 5.8^{\mathrm{bc}}$ \\
\hline
\end{tabular}

${ }^{z}$ Puerarin content is given in milligrams per gram of $20 \%$ ethanol extract.

${ }^{\mathrm{y}}$ The values represent the mean \pm standard error, and the mean values with a different letter are significantly different at $\mathrm{P}<0.05$.

Table 4 Total contents of daidzin and daidzein in the hairy root for 2 arrowroot lines. The experiment was conducted 6 times over a 4-week period

\begin{tabular}{ccccc}
\hline & 1 week & 2 weeks & 3 weeks & 4 weeks \\
\hline $\mathrm{DJ}^{\mathrm{z}, \mathrm{y}}$ & $6.9 \pm 0.9^{\mathrm{bc}}$ & $5.5 \pm 1.0^{\mathrm{cd}}$ & $6.0 \pm 0.7^{\mathrm{cd}}$ & $7.4 \pm 0.7^{\mathrm{bc}}$ \\
$\mathrm{DY}^{\mathrm{z}, \mathrm{y}}$ & $12.8 \pm 1.3^{\mathrm{a}}$ & $9.1 \pm 1.1^{\mathrm{b}}$ & $5.5 \pm 0.8^{\mathrm{cd}}$ & $3.9 \pm 0.9^{\mathrm{d}}$ \\
\hline
\end{tabular}

${ }^{\mathrm{z}}$ Total content of daidzin and daidzein is given in milligrams per gram of $20 \%$ ethanol extract.

${ }^{\mathrm{y}}$ The values represent the mean \pm standard error, and the mean values with a different letter are significantly different at $\mathrm{P}<0.05$.

Table 5 Daily production of puerarin in the hairy root for 2 arrowroot lines. The experiment was conducted 6 times over a 4-week period

\begin{tabular}{ccccc}
\hline & 1 week & 2 weeks & 3 weeks & 4 weeks \\
\hline $\mathrm{DJ}^{\mathrm{z}, \mathrm{y}}$ & $1.876 \pm 0.309^{\mathrm{bc}}$ & $1.846 \pm 0.405^{\mathrm{bc}}$ & $1.985 \pm 0.218^{\mathrm{bc}}$ & $2.200 \pm 0.420^{\mathrm{bc}}$ \\
$\mathrm{DY}^{\mathrm{z}, \mathrm{y}}$ & $3.951 \pm 0.321^{\mathrm{a}}$ & $2.643 \pm 0.231^{\mathrm{b}}$ & $1.861 \pm 0.408^{\mathrm{bc}}$ & $1.199 \pm 0.515^{\mathrm{c}}$ \\
\hline
\end{tabular}

${ }^{\mathrm{z}}$ Average production of puerarin is given in milligrams per gram of $20 \%$ ethanol extract and day.

${ }^{\mathrm{y}}$ The values represent the mean \pm standard error, and the mean values with a different letter are significantly different at $\mathrm{P}<0.05$.

Table 6 Daily production of daidzin and daidzein in the hairy root for the 2 arrowroot lines. The experiment was conducted 6 times over a 4-week period

\begin{tabular}{ccccc}
\hline & 1 week & 2 weeks & 3 weeks & 4 weeks \\
\hline $\mathrm{DJ}^{\mathrm{z}, \mathrm{y}}$ & $0.345 \pm 0.075^{\mathrm{bc}}$ & $0.311 \pm 0.085^{\mathrm{bc}}$ & $0.352 \pm 0.047^{\mathrm{bc}}$ & $0.436 \pm 0.091^{\mathrm{bc}}$ \\
$\mathrm{DY}^{\mathrm{z}, \mathrm{y}}$ & $1.321 \pm 0.156^{\mathrm{a}}$ & $0.600 \pm 0.083^{\mathrm{b}}$ & $0.386 \pm 0.072^{\mathrm{bc}}$ & $0.219 \pm 0.089^{\mathrm{c}}$ \\
\hline
\end{tabular}

${ }^{\mathrm{z}}$ The mean production of daidzin and daidzein is given in milligrams per gram of $20 \%$ ethanol extract and day.

${ }^{\mathrm{y}}$ The values represent the mean \pm standard error, and the mean values with a different letter are significantly different at $\mathrm{P}<0.05$.

from the hairy roots harvested each week were analyzed (Table 3, 4). The differences in both the puerarin and daidzin+daidzein contents between DJ7 and DY1 were significant at $\mathrm{P}<0.05$, and those depending on the culture period were also significantly different. The highest mean puerarin contents in DJ7 and DY1 were 39.0 and $40.5 \mathrm{mg} / \mathrm{g}$ of $20 \%$ ethanol extracts on the $1^{\text {st }}$ and $2^{\text {nd }}$ week of culture, respectively, whereas those of daidzin+daidzein were 7.4 and $12.8 \mathrm{mg} / \mathrm{g}$ of $20 \%$ ethanol extracts on the $4^{\text {th }}$ and $1^{\text {st }}$ week of culture, respectively. When the two lines were analyzed separately, both the puerarin and daidzin+daidzein contents measured each week were not significantly different in DJ7. On the other hand, the levels of both decreased gradually after one or two weeks in DY1. These results show a similar pattern of isoflavone accumulation in the $P$. candollei hairy root cultures (Udomsuk et al. 2011).
The daily production of both puerarin and daidzin+daidzein in 20\% ethanol extracts from the hairy roots harvested every week were also analyzed (Table 5,6). The highest mean daily production of puerarin in DJ7 and DY1 were 2.200 and $3.951 \mathrm{mg} / \mathrm{g}$ of $20 \%$ ethanol extracts and day on the $4^{\text {th }}$ and $1^{\text {st }}$ week of culture and the corresponding level of daidzin+daidzein were 0.436 and $1.321 \mathrm{mg} / \mathrm{g}$ of $20 \%$ ethanol extract and day on $4^{\text {th }}$ and $1^{\text {st }}$ week of culture, respectively. When the two lines were analyzed separately, the daily production of both puerarin and daidzin+daidzein measured each week were similar in DJ7. On the other hand, the levels of both puerarin and daidzin+daidzein in DY1 decreased with time.

Overall, the results regarding the growth of hairy roots and isoflavone accumulation in hairy roots showed that a culture period of one or two weeks would be the best for producing the isoflavones efficiently in vitro. 
Table 7 Puerarin, daidzin, daidzein and dihydrodaidzein contents after fermentation of the hairy root extracts of 2 Korean wild arrowroot with $P$. pentosaceus EP108

\begin{tabular}{|c|c|c|c|c|c|}
\hline Line & Time(hours) & Puerarin & Daidzin & Daidzein & DHD \\
\hline \multirow{4}{*}{$\mathrm{DJ} 7^{\mathrm{z}, \mathrm{y}}$} & 0 & $0.94 \pm 0.00$ & $9.11 \pm 0.02$ & $199.87 \pm 1.14$ & $\mathrm{ND}^{\mathrm{x}}$ \\
\hline & 24 & $0.31 \pm 0.01$ & $6.52 \pm 0.15$ & $157.60 \pm 4.03$ & ND \\
\hline & 48 & $0.42 \pm 0.06$ & $0.36 \pm 0.03$ & $13.33 \pm 6.90$ & $5.40 \pm 0.46$ \\
\hline & 72 & $2.91 \pm 0.56$ & ND & $0.47 \pm 0.01$ & $5.40 \pm 0.35$ \\
\hline \multirow{4}{*}{$D Y 1^{z, y}$} & 0 & $1.11 \pm 0.07$ & $7.71 \pm 0.09$ & $64.75 \pm 1.62$ & ND \\
\hline & 24 & $0.98 \pm 0.00$ & $4.11 \pm 0.02$ & ND & ND \\
\hline & 48 & $2.77 \pm 0.01$ & $0.29 \pm 0.01$ & ND & $\mathrm{ND}$ \\
\hline & 72 & $3.23 \pm 0.25$ & $0.12 \pm 0.01$ & ND & ND \\
\hline
\end{tabular}

${ }^{\mathrm{z}}$ Contents of puerarin, daidzin, daidzein and DHD are given in milligrams per gram of $20 \%$ ethanol extract.

${ }^{\mathrm{y}} 0,24,48$ and 72 indicate the fermentation time with $P$. pentosaceus EP106.

${ }^{\mathrm{x}} \mathrm{ND}$ indicates 'not detected'.

Biotransformation of isoflavones

To examine the biotransformation of isoflavones in vitro, the hairy root extracts of two Korean wild arrowroots (DJ7 and DY1) were fermented with $P$. pentosaceus EP108 which has been identified to have ability to biotransform isoflavones (unpublished data). Equol precursors, such as puerarin, daidzin, daidzein and dihydrodaidzein, were analyzed depending on the fermentation period, 0, 24, 48 and 72 hours. Comparing with initial contents of puerarin and daidzein in callus previously reported by Lee et al. (2013), puerarin was less, but daidzein was more in hairy root before fermentation. After fermentation, in the case of DJ7, dihydrodaidzein was detected in the extracts co-cultured with the strain for 48 and 72 hours, but wasn't produced in DY1 until 72 hours (Table 7). These results show that daidzin and daidzein have a decreasing tendency with increasing reaction time. However, the rate of biotransformation in hairy root was lower than that in callus. The results obtained in this study suggest that daidzin and daidzein are converted to other isoflavone metabolites including dihydrodaidzein and/or equol by the bio-conversion capability of the intestinal bacteria (Jin et al. 2008; Lee et al. 2013; Park et al. 2006; Shimada et al. 2010; Tsuji et al. 2012).

In conclusion, this study confirmed the growth hairy roots in vitro and isoflavone production in the hairy root of Korean wild arrowroot and dihydrodaidzein biosynthesis via the fermentation of a hairy root extract with $P$. pentosaceus. These results are expected to facilitate the mass production of hairy roots and isoflavones as equol precursors from Korean wild arrowroot, and will form the foundation for the production of equol by biotransformation in vitro. Finally, the overall aim of this project was the mass production of equol via biotransformation in vitro. Therefore, the following additional studies will be needed: the enhancement of biomass and effective mass production of the isoflavone from $P$. tuberosa using a bioreactor (Sharma et al. 2009), enhancement of the isoflavone content of $P$. candollei by abiotic and biotic elicitor treatments such as methyl jasmonate, chitosan, yeast extract, etc. (Korsangruang et al. 2010), and the confirmation of equol production by the reaction of hairy root extract with the strain used in this study.

\section{Acknowledgments}

This study was supported by the Daegu University Research Grant, 2015.

\section{References}

Arai Y, Uehara M, Sato Y, Kimira M, Eboshida A, Adlercreutz H, Watanabe S (2000) Comparison of isoflavones among dietary intake, plasma concentration and urinary excretion for accurate estimation of phytoestrogen intake. J Epidemiol 10:127-135

Barnes S (1998) Evolution of the health benefits of soy isoflavones. Proc Soc Exp Biol Med 217:386-392

Boonsnongcheep P, Korsangruang S, Soonthornchareonnon N, Chintapakorn Y, Saralamp P, Prathanthurarug S (2010) Growth and isoflavonoid accumulation of Pueraria candollei var. candollei and $P$. candollei var. mirifica cell suspension cultures. Plant Cell Tiss Org 101:119-126

Bourgaud F, Bouque V, Guckert A (1999) Production of flavonoids by Psoralea hairy root cultures. Plant Cell Tiss Org 56:97-104

Cassidy A (1996) Physiological effects of phyto-oestrogens in relation to cancer and other human health risks. Proc Nutr Soc 55:399-417

Chansakaow S, Ishikawa T, Sekine K, Okada M, Higushi Y, Kudo M, Chaichantipyuth C (2000) Isoflavonoids from Pueraria 
mirifica and their estrogenic activity. Planta Med 66:572-574

Chen G, Li L (2007) Nutrient consumption and production of isoflavones in bioreactor cultures of Pueraria lobata (Willd). J Environ Biol 28:321-326

Cherdshewasart W, Subtang S, Dahlan W (2007) Major isoflavonoid contents of the phytoestrogen rich-herb Pueraria mirifica in comparison with Pueraria lobata. J Pharm Biomed Anal 43:428-434

Decroos K, Vanhemmens S, Cattoir S, Boon N, Verstraete, W (2005) Isolation and characterisation of an equol-producing mixed microbial culture from a human faecal sample and its activity under gastrointestinal conditions. Arch Microbiol 183:45-55

Jin J-S, Nishihata T, Kakiuchi N, Hattori M (2008) Biotransformation of C-glucosylisoflavone Puerarin to Estrogenic (3S)-equol in co-culture of two human intestinal bacteria. Biol Pharm Bull 31:1621-1625

Junko H, Naoki N, Keiichi U (1984) Studies on physical and chemical quality evaluation of crude drug preparations. YakugakuZasshi 104:50-56

Kaufman PB, Duke JA, Brielmann H, Boik J, Hoyt JE (1997) A comparison survey of leguminous plants as sources of the isoflavones, genistein and daidzein: implications for human nutrition and health. J Altern Complement Med 3:7-12

Kim DK, Jang DS, Kim JH (2009) Genetic variations and phylogenetic relationship of and Pueraria lobata Ohwi (Fabaceae) and related Taxa by RAPD makers. Kor J Plant Res 22: 446-453

Kim MH, Park SB (1987) Studies on the content of Pueraria radix in the tea by HPLC. Kor J Food Hygiene 2:89-95

Kim S, Cha M-S, Lee E, Kim I, Kwon JE, Kang SC, Park T-H (2012) In vitro induction of hairy root from isoflavonesproducing Korean wild arrowroot Pueraria lobata. J Plant Biotechnol 39:205-211

Korsangruang S, Soonthornchareonnon N, Chintapakorn Y, Saralamp P, Prathanturarug S (2010) Effects of abiotic and biotic elicitors on growth and isoflvonoid accumulation in Pueraria candollei var. candollei and $P$. candollei var. mirifica cell suspension cultures. Plant Cell Tiss Org 103:333-342

Lee E, Kwon JE, Cha M-S, Kim I, Kang SC, Park T-H (2013) Isoflavones and biotransformed dihydrodadizein production with in vitro cultured callus of Korean wild arrowroot Pueraria lobata. J Plant Biotechnol 40:217-223

Minamida K, Tanaka M, Abe A, Sone T, Tomita F, Hara H, Asano K (2006) Production of equol from daidzein by gram-positive rod-shaped bacterium isolated from rat intestine. J Biosci Bioeng 102:247-250

Murashige T, Skoog F (1962) A revised medium for rapid growth and bioassays with tobacco tissue culture. Physiol Plant 15:473-479

Oh M-J, Lee K-S, Son H-Y, Kim S-Y (1990) Antioxidative compounds of Pueraria root. Kor J Food SciTechnol 22:793-198

Park E-K, Shin JS, Bae E-A, Lee Y-C, Kim D-H (2006) Intestinal bacteria activate estrogenic effect of main constituents puerarin and daidzin of Pueraria thunbergiana. Biol Pharm Bull 29:2432-2435

Sathyamoorthy N, Wang TT (1997) Differential effects of dietary phy-to-estrogens daidzein and equol on human breast cancer MCF-7 cells. Eur J Cancer 33:2384-2389

Setchell KDR (1998) Phytoestrogens: the biochemistry, physiology, and implications for human health of soy isoflavones. Am J Clin Nutr 68:1333S-1346S

Setchell KDR, Brown NM, Lydeking-Olsen E (2002) The clinical importance of the metabolite equol - A clue to the effectiveness of soy and its isoflavones. J Nutr 132:3577-3584

Setchell KDR, Brown NM, Summer S, King EC, Heubi JE, Cole S, Guy T, Hokin B (2013) Dietary factors influence production of the soy isoflavone metabolite S-(-)equol in healthy adults. J Nutr 143:1950-1958

Sharma V, Goyal S, Ramawat KG (2009) Scale up production of isoflavonoids in cell suspension cultures of Pueraria tuberosa grown in shake flasks and bioreactor. Eng Life Sci 9:267-271

Shimada Y, Yasuda S, Takahashi M, Hayashi T, Miyazawa N, Sata I, Abiru Y, Uchiyama S, Hishigaki H (2010) Cloning and expression of a novel NADP(H)-dependent daidzein reductase, an enzyme involved in the metabolism of daidzein, from equol-producing Lactococcus strain 20-92. Appl Environ Microb 76:5892-5901

Shin SC, Kang SK, Jang MJ (2000) A study on the screening of antimicrobial activity and the isoflavonoids in Korean arrowroot. Kor J Plant Res 13:25-28

Spennemann DHR (1992) Arrowroot production in the Marshall islands: past, present, and future. New Zealand J Crop Hort 20:97

Spennemann DHR (1994) Traditional arrowroot production and utilization in the Marshall islands. J Ethnobiol 14:211-234

Tsuji H, Moriyama K, Nomoto K, Akaza H (2012) Identification of an enzyme system for daidzein-to-equol conversion in Slakia sp. Strain NATTS. Appl Environ Microb 78:1228-1236

Udomsuk L, Jarukamjorn K, Tanaka H, Putalun W (2011) Improved isoflavonoid production in Pueraria candollei hairy root cultures using elicitation. Biotechnol Lett 33:369-374

Vaishnav K, Goyal S, Ramawat KG (2006) Isoflavonoids production in callus culture of Pueraria tuberosa, the Indian Kudzu. Indian J Exp Biol 44:1012-1017

Watanabe S, Terashima K, Sato Y, Arai S, Eboshida A (2000) Effects of isoflavone supplement on healthy women. Biofactors 12:233-241

Yokoyama S, Suzuki T (2008) Isolation and characterization of a novel equol-producing bacterium from human feces. Biosci Biotechnol Biochem 72:2660-2666

Yu Z-T, Yao W, Zhu W-Y (2008) Isolation and identification of equol-producing bacterial strains from cultures of pig faeces. FEMS Microbiol Lett 282:73-80

Yuk CS (1989) Colored medicinal plants of Korea. Academy book, Seoul 